Bio - grafía. Escritos sobre la Biología y su Enseñanza. ISSN 2027-1034

Edición Extraordinaria. p.p. 1423 - 1432

Memorias del IX Encuentro Nacional de Experiencias en Enseñanza de la Biología y la

Educación Ambiental. IV Congreso Nacional de Investigación en Enseñanza de la Biología.

\title{
EVALUACIÓN ALTERNATIVA Y HERRAMIENTAS DE EVALUACIÓN EN LA ENSEÑANZA DEL CONCEPTO "RESPIRACIÓN"
}

\author{
Velásquez Almonacid, Juan Nicolas ${ }^{1}$ \\ Rey Pulido, Karina Gise $I^{2}$ \\ Villanueva Solano, David Fernando ${ }^{3}$
}

\section{RESUMEN}

El presente artículo muestra las implicaciones del uso de diferentes herramientas evaluativas durante el proceso de enseñanza-aprendizaje del concepto respiración en seres vivos. Para esto se diseñaron dos instrumentos de evaluación; uno de ellos el cual denominamos cuestionario estructurado, el cual ofrecía a los estudiantes una serie de preguntas cerradas, las cuales podían resolverse mediante el uso de bancos de respuestas obtenidos de medios externos (internet, literatura relacionada o indagación a terceros). El segundo instrumento es la elaboración de mapas conceptuales pues al ser una herramienta no limitante, permite una interacción más flexible entre conceptos lo cual refleja una clara observación de los posibles problemas en el proceso enseñanzaaprendizaje. Como principal resultado se evidencia que la herramienta evaluativa que más aporta en este tipo de evaluación son los mapas conceptuales. respiración.

PALABRAS CLAVE: Mapas conceptuales, cuestionario, evaluación alternativa,

ABSTRACT: The present article shows the implications of using different evaluative tools during the teaching-learning process of the concept of breathing in living beings. Two evaluation instruments were designed; one of them is called a structured questionnaire, which offers students a set of closed questions, which could be solved by the use of external response banks (internet, related literature or third-party inquiry). The second instrument is the development of conceptual maps for a non-limiting tool, allows for a more flexible interaction between concepts for which it reflects a clear observation of possible problems in the teaching-learning process. As the main result, it is evident that the evaluative tool that contributes most to this type of evaluation of the concept maps.

\footnotetext{
1 Estudiantes Licenciatura en Biología Universidad Distrital Francisco José de Caldas. icolasvelasquez88@hotmail.com

${ }^{2}$ Estudiantes Licenciatura en Biología Universidad Distrital Francisco José de Caldas. karinagisel410@hotmail.com

${ }^{3}$ Estudiantes Licenciatura en Biología Universidad Distrital Francisco José de Caldas. david.villa93@outlook.com
} 
Bio - grafía. Escritos sobre la Biología y su Enseñanza. ISSN 2027-1034

Edición Extraordinaria. p.p. 1423 - 1432

Memorias del IX Encuentro Nacional de Experiencias en Enseñanza de la Biología y la

Educación Ambiental. IV Congreso Nacional de Investigación en Enseñanza de la Biología.

KEY WORDS: Conceptual maps, questionnaire, alternative assessment, breathing.

\section{INTRODUCCIÓN:}

Los principales estatutos y códigos que regulan el sistema de educación en Colombia, han logrado diversificar el concepto de evaluación llevándola a campos mucho más amplios, ya no se busca una revisión del rendimiento académico de los estudiantes únicamente sino que se pretende lograr una revisión globalizada del aprendizaje, del funcionamiento de las instituciones educativas y de las prácticas docentes. Es importante destacar la relación estrecha e interconectada que presenta la educación y la evaluación, de hecho su relación ha redefinido el diseño de políticas públicas, sin embargo hace falta un sistema propio de evaluación que llegue en sus resultados al objetivo real de lo que se busca con una evaluación, por tanto surgen movimientos y posturas como la evaluación alternativa, que justamente quiere proponer nuevos métodos, herramientas e instrumentos de evaluación, donde el estudiante plasme la conexión de su aprendizaje con su contexto, y se permita identificar por medio de ésta los posibles errores que se presentan en el proceso enseñanza-aprendizaje.

Así se inicia este proceso de exploración en la pertinencia de instrumentos de evaluación a la hora de brindar un diagnóstico más efectivo que permita tomar decisiones frente a los resultados obtenidos, para mejorar el proceso enseñanza-aprendizaje. Para ello, se realizó una intervención en la Institución Educativa Distrital República de Colombia, en el grado 702 conformado por 39 estudiantes de edades entre los 12 y los 14 años. Se diseñaron dos instrumentos de evaluación, el primero consiste en la elaboración de un mapa conceptual del proceso de respiración en animales y plantas, el otro instrumento consistía en cuestionarios estructurado referente a la respiración; éstas herramientas tienen como objetivo diagnosticar los posibles problemas que se presentan en el proceso de enseñanza-aprendizaje del concepto respiración en seres vivos, y de éste modo analizar cuál de los dos instrumentos da cuenta de éstos posibles problemas.

Dicha institución presenta dentro de su PEI, el enfoque de aprendizaje significativo como marco teórico de referencia para los procesos de enseñanzaaprendizaje de los estudiantes. Es por esto que se ve la necesidad de abordar los planteamientos que menciona el aprendizaje significativo. Así pues se entiende que el objetivo base del aprendizaje significativo es que los estudiantes lleguen a una comprensión tan auténtica que logren de forma autónoma descubrir por medio de los nuevos conocimientos los errores que tenían frente a un tema determinado y que se haga una cohesión entre éstos nuevos aprendizajes y las ideas previas acertadas existentes en su sistema cognitivo (Ausubel, 1973, 1976, 2002).

Desde la perspectiva del aprendizaje significativo, nace la implementación de herramientas que den cuenta de dicho aprendizaje, es así como surge la elaboración de mapas conceptuales como método de evaluación para el aprendizaje significativo. Por consiguiente, consistirán en la unión de dos o más palabras por medio de un conector 
Bio - grafía. Escritos sobre la Biología y su Enseñanza. ISSN 2027-1034

Edición Extraordinaria. p.p. 1423 - 1432

Memorias del IX Encuentro Nacional de Experiencias en Enseñanza de la Biología y la

Educación Ambiental. IV Congreso Nacional de Investigación en Enseñanza de la Biología.

para conformar una proposición (Novak y Gowin, 1998). Por consiguiente, el cuestionario estructurado siendo una herramienta de sencillo y amplio uso, que posibilita evaluar cuantitativamente los procesos de enseñanza aprendizaje desarrollados en el aula, fue el segundo instrumento utilizado en el presente escrito.

\section{Aprendizaje significativo}

El aprendizaje significativo, propuesto teórico de Ausubel (1973, 1976, 2002) menciona a la estructura cognitiva del alumno como el conjunto de ideas y conceptos previos que posee frente a un área del conocimiento particular y que resulta de suma importancia pues permite que de la confrontación con el nuevo conocimiento el alumno afiance y robustezca lo que aprende a partir de una constante complementación. En síntesis, el aprendizaje significativo ocurre cuando una nueva información se conecta con un concepto existente en la estructura cognitiva de los estudiantes.

\section{Evaluación alternativa}

La aplicación de nuevas estrategias de evaluación se hace evidente por la existencia de un espectro mucho más amplio de desempeño que los estudiantes pueden mostrar. Ahumada (2005) Con base en tal desempeño surge la evaluación alternativa, cuya razón es indagar qué sabe el estudiante, cómo asume y sitúa en su contexto particular el conocimiento construido desde sus ideas previas; lo cual exige desarrollar diversas estrategias evaluativas. El sustento teórico que apoya esta nueva corriente de evaluación alternativa se sienta en las concepciones de aprendizaje significativo propuestas por Ausubel (1976). Para Ausubel (como se citó en Rodríguez, 2004) esta teoría aborda íntegramente, todos y cada uno de los factores, componentes y requisitos que certifican la adquisición, y asimilación del entramado de contenido que ofrece la escuela a sus estudiantes de tal forma que adquiera significado para el mismo. Ahora bien retomando la intencionalidad que presenta la evaluación alternativa, es importante mencionar que uno de sus objetivos es que el estudiante logre una responsabilidad de su propio aprendizaje y qué éste proceso sirva como medio para lograr afianzar sus conocimientos.

Durante un proceso evaluativo centrado en el aprendizaje debe ser esencial que se logre establecer niveles de avance o progreso en el acercamiento a un determinado conocimiento, tomando su incorporación significativa o su relación con los conocimientos previos que posee cada estudiante. Ahumada (2005) menciona como resultado de éste proceso el profesor se ve obligado a estar siempre atento a las posibles dificultades que sufren los diferentes procesos de enseñanza-aprendizaje, para hacer las observaciones y correcciones pertinentes y que logre en el estudiante un reconocimiento discutible de sus relaciones conceptuales.

\section{Respiración en seres vivos}

El proceso de respiración es llevado a cabo por los organismos aerobios, ya que para ellos es absolutamente necesario el oxígeno para la realización de sus actividades 
Bio - grafía. Escritos sobre la Biología y su Enseñanza. ISSN 2027-1034

Edición Extraordinaria. p.p. 1423 - 1432

Memorias del IX Encuentro Nacional de Experiencias en Enseñanza de la Biología y la

Educación Ambiental. IV Congreso Nacional de Investigación en Enseñanza de la Biología.

metabólicas a nivel celular. Es así como los diferentes organismos, presentan diferentes sistemas de hematosis, bien sea cutáneo, traqueal, branquial entre otros. Como residuo de estos procesos se obtiene un producto de desecho llamado dióxido de carbono, el cuál es devuelto al medio exterior dependiendo el sistema de intercambio que presente. El oxígeno que ingresa a los diferentes organismos, bien sean plantas o animales, es transformado a nivel celular en energía química que es imprescindible en todos los procesos vitales, como bien se plantea el objetivo esencial de la respiración, es obtener energía luego de varias reacciones químicas a nivel celular (Curtis, Barnes, Schnek y Flores, 2006).

En el campo de la enseñanza del tema respiración, se observan diferentes concepciones presentes en los estudiantes a la hora de expresar su idea sobre respiración, una de las más frecuentes es que se determina éste proceso a un mero intercambio de gases (Orrego, Dávila y Tamayo, 2009). Para Hatano y Inagaki (lbid., p, 1821) otro modelo explicativo que es común en los estudiantes, es el que se trasporta de un plano vitalista a una visión mecanicista de lo que es la respiración. También Königsberg (lbid., p. 1821) resalta la importancia del dibujo animado como instrumento de explicación para éste concepto y en general para la construcción del pensamiento científico y como una buen método para la identificación de obstáculos en el proceso enseñanza-aprendizaje del concepto de respiración.

\section{Instrumentos de Evaluación}

Hoy en día varios instrumentos y técnicas son empleados a la hora de realizar una evaluación para recolectar evidencia del aprendizaje de los estudiantes y de los problemas durante éste proceso, dichos instrumentos pueden ser: mapas semánticos y conceptuales, la UVE de Gowin y los gráficos de síntesis de prácticas como la elaboración de organigramas, ideogramas, flujogramas, etc (Ahumada, 2005). El mapa conceptual es un buen instrumento para la evaluación alternativa, ya que confiere ayuda a los estudiantes en el momento de evidenciar sus concepciones pues propone una imagen clara de los conceptos y preposiciones que han apropiado en su estructura cognitiva; razón por la cual es efectivo. Son asimismo instrumentos adecuados en la medida que revelan las concepciones difusas, llegando a ser denotadas por el nexo que conforman dos conceptos que forman una preposición notoriamente falsa, o bien sea por un nexo que deja en manifiesto el paso por alto de la idea principal que relaciona dos o más conceptos, teniendo en cuenta igualmente que los alumnos no son una tabla rasa donde hay que escribir o un depósito vac ío que deba llenarse (Novak y Gowin, 1988).

Por otro lado los cuestionarios, son instrumentos de evaluación clásicos que consisten en un conjunto de preguntas, normalmente de varios tipos, diseñado de forma cuidadosa para obtener resultados en su mayoría de forma cuantitativa, respecto a un tema de evaluación. El diseño de un cuestionario presenta preguntas cerradas o abiertas; en la primera, el estudiante que va a ser evaluado tiene toda una gama de alternativas posibles que dan respuesta a la situación presentada, el estudiante no tiene sino que elegir alguna o algunas respuestas de las cuales se presentan. Según la naturaleza del 
Bio - grafía. Escritos sobre la Biología y su Enseñanza. ISSN 2027-1034

Edición Extraordinaria. p.p. 1423 - 1432

Memorias del IX Encuentro Nacional de Experiencias en Enseñanza de la Biología y la

Educación Ambiental. IV Congreso Nacional de Investigación en Enseñanza de la Biología.

contenido de las preguntas cerradas estas pueden ser: de hecho, de opinión, de identificación, de acción, de información y de intención. Mientras que las preguntas abiertas son más fáciles de formular, puesto que no hay que prever ningún tipo de respuesta. Sin embargo se delimita con un enunciado al cual deben dar respuesta concreta desde los nuevos conocimientos que ha logrado cohesionar con sus ideas previas (Muñoz, 2003).

\section{METODOLOGÍA}

El actual trabajo se desarrolló en la Institución Educativa Distrital Colegio República de Colombia con estudiantes del grado 702. Semanalmente a la asignatura Biología le fueron convenidos los días miércoles y viernes con una duración respectiva de una hora, por consiguiente este trabajo se desarrolló en tres (3) fases que permitieron organizar cronológicamente las acciones en el aula:

\section{a. Revisión bibliográfica sobre posibles obstáculos en la enseñanza- aprendizaje del concepto "respiración"}

Orrego, Dávila \& Tamayo (2009) y, Monguí (2014), sugieren una serie de obstáculos en el proceso de aprendizaje del concepto "respiración" los cuales deben ser identificados y son de vital importancia en el momento de desarrollar diferentes tipos de estratégicas didáctico-pedagógicas a futuro en pro del correcto entendimiento del concepto "respiración". A continuación rescatamos algunos de estos posibles obstáculos presentes en el aula:

1. Confusión con la fotos íntesis.

2. Excesivo antropocentrismo.

3. No se hace referencia a respiración celular.

4. Desarticular la respiración de otros procesos celulares y del organismo.

5. Dificultad en el uso del lenguaje especializado.

(lbid., p. 1822) (Monguí. Op. cit, p. 82)

\section{b. Aplicación de herramientas evaluativas: Mapas conceptuales y cuestionarios}

La herramienta de mapas conceptuales se abordó mediante la elaboración de un 1 punto, el cual consistió en el desarrollo de un mapa conceptual sobre respiración en animales y respiración en plantas. Según Novak y Gowin (como se citó en Mendonça y 
Bio - grafía. Escritos sobre la Biología y su Enseñanza. ISSN 2027-1034

Edición Extraordinaria. p.p. 1423 - 1432

Memorias del IX Encuentro Nacional de Experiencias en Enseñanza de la Biología y la

Educación Ambiental. IV Congreso Nacional de Investigación en Enseñanza de la Biología.

Silveira, 2012) estos mapas conceptuales, cuando se utilizan como herramienta de evaluación del aprendizaje, son una potente fuente de información del proceso de aprendizaje del alumno, para eso es primordial analizar los conceptos involucrados y las relaciones entre ellos; para el caso del cuestionario, se articuló con una serie de preguntas extraídas de bancos de preguntas en fuentes externas, su contenido se desarrolla a través de 4 puntos a resolver; 3 preguntas de opción múltiple y 1 esquema para completar del sistema respiratorio, desarrollados con el fin de dar a los estudiantes, un margen de error nulo para su respuesta. En la tabla 1 incluimos los resultados obtenidos dentro de la matriz diseñada para el seguimiento de los posibles obstáculos presentes en el aprendizaje del concepto respiración.

\section{c. Recolección de datos suministrados por las herramientas evaluativas}

Teniendo en cuenta estas especificaciones y los posibles obstáculos en el aprendizaje del concepto respiración se diseñó una tabla, la cual ayudó a esquematizar el proceso de evaluación de una forma más organizada. A continuación en las figuras 1 y 2 se muestra un ejemplo tanto de mapas conceptuales como del cuestionario diseñado en este trabajo. Se eligieron 12 estudiantes para el tratamiento de sus evaluaciones pues mostraron constancia en su responsabilidad formativa durante las sesiones de clase.

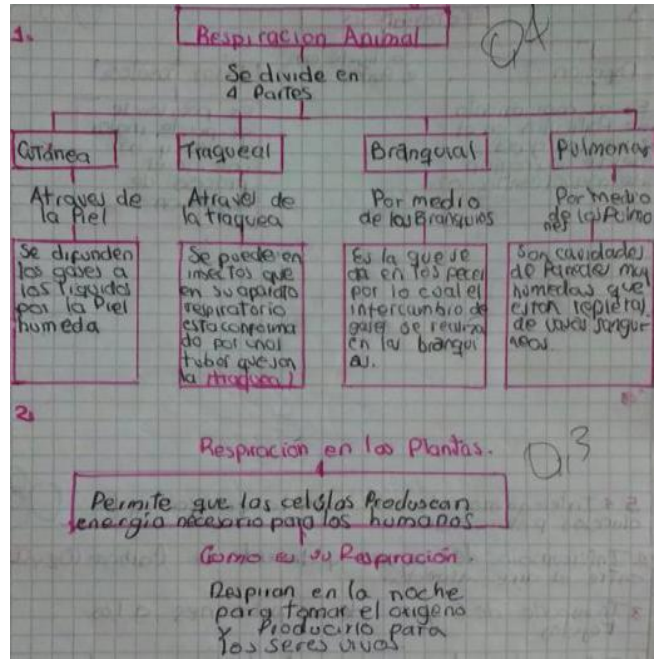

Figura 1. Mapa conceptual: En el presente mapa conceptual evidenciamos lo obstáculos: Confusión con la fotosíntesis, No se hace referencia a respiración celular, Desarticular la respiración de otros procesos celulares y del organismo, Dificultad en el uso del lenguaje especializado.

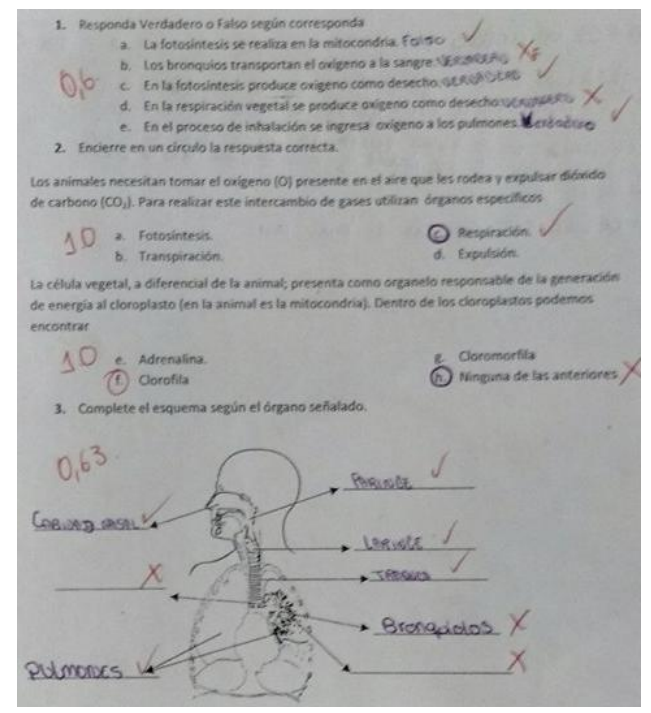

Figura 2. Cuestionario: En el presente cuestionario evidenciamos los obstáculos: Dificultad en el uso del lenguaje especializado. 
Bio - grafía. Escritos sobre la Biología y su Enseñanza. ISSN 2027-1034

Edición Extraordinaria. p.p. 1423 - 1432

Memorias del IX Encuentro Nacional de Experiencias en Enseñanza de la Biología y la Educación Ambiental. IV Congreso Nacional de Investigación en Enseñanza de la Biología.

\section{RESULTADOS:}

Dentro de la siguiente tabla se encuentra el conteo de los obstáculos evidenciados dentro de cada una de las herramientas. Para las primeras cinco (5) columnas que representan los posibles obstáculos el uno (1) representa presencia del obstáculo, mientras el cero (0) representa ausencia del obstáculo. Para las dos siguientes se muestra un conteo individual tanto de conceptos como de enlaces cruzados para evidenciar indicios de aprendizaje significativo. El patrón se repite para incluir los resultados de la segunda herramienta diseñada.

Tabla 1. Se muestra de forma cuantitativa el número de obstáculos evidenciados además de evidencias de un aprendizaje significativo a través del análisis de los mapas conceptuales y cuestionarios generados por los estudiantes. Se muestra a: $\mathrm{CF}=$ Confusión con la fotosíntesis, $\mathrm{EA}=$ Excesivo antropocentrismo, $\mathrm{NRC}=$ No se hace referencia a respiración celular, DRP = Desarticular la respiración de otros procesos celulares y del organismo, DLE= Dificultad en el uso del lenguaje especializado.

\begin{tabular}{|c|c|c|c|c|c|c|c|c|c|c|c|c|c|c|}
\hline & \multicolumn{9}{|c|}{ MAPAS CONCEPTUALES } & \multicolumn{6}{c|}{ CUESTIONARIOS } \\
\cline { 2 - 13 } & $\begin{array}{c}\text { C } \\
\text { A }\end{array}$ & $\begin{array}{c}\text { NR } \\
\text { C }\end{array}$ & $\begin{array}{c}\text { DR } \\
\text { P }\end{array}$ & $\begin{array}{c}\text { D } \\
\text { EL }\end{array}$ & $\begin{array}{c}\text { Conce } \\
\text { ptos }\end{array}$ & $\begin{array}{c}\text { Conect } \\
\text { ores }\end{array}$ & $\begin{array}{c}\text { C } \\
\text { F }\end{array}$ & $\begin{array}{c}\text { E } \\
\text { A }\end{array}$ & $\begin{array}{c}\text { NR } \\
\text { C }\end{array}$ & $\begin{array}{c}\text { DR } \\
P\end{array}$ & $\begin{array}{c}\text { D } \\
\text { EL }\end{array}$ & $\begin{array}{c}\text { Conce } \\
\text { ptos }\end{array}$ & $\begin{array}{c}\text { Conect } \\
\text { ores }\end{array}$ \\
\hline $\begin{array}{c}\text { Caso } \\
2\end{array}$ & 1 & 1 & 1 & 1 & 1 & 7 & 3 & 0 & 0 & 0 & 0 & 0 & N/A & N/A \\
\hline $\begin{array}{c}\text { Caso } \\
3\end{array}$ & 0 & 1 & 1 & 1 & 1 & 6 & 3 & 0 & 0 & 0 & 0 & 0 & N/A & N/A \\
\hline $\begin{array}{c}\text { Caso } \\
4\end{array}$ & 1 & 1 & 1 & 1 & 1 & 14 & 3 & 0 & 0 & 0 & 0 & 0 & N/A & N/A \\
\hline $\begin{array}{c}\text { Caso } \\
5\end{array}$ & 1 & 1 & 1 & 1 & 1 & 10 & 1 & 0 & 0 & 0 & 0 & 0 & N/A & N/A \\
\hline $\begin{array}{c}\text { Caso } \\
6\end{array}$ & 1 & 1 & 1 & 1 & 1 & 7 & 3 & 0 & 0 & 0 & 0 & 0 & N/A & N/A \\
\hline $\begin{array}{c}\text { Caso } \\
7\end{array}$ & 0 & 1 & 1 & 1 & 1 & 6 & 0 & 0 & 0 & 0 & 1 & 1 & N/A & N/A \\
\hline $\begin{array}{c}\text { Caso } \\
8\end{array}$ & 1 & 1 & 1 & 1 & 1 & 6 & 0 & 0 & 0 & 0 & 0 & 0 & N/A & N/A \\
\hline $\begin{array}{c}\text { Caso } \\
9\end{array}$ & 1 & 0 & 1 & 1 & 1 & 13 & 4 & 1 & 0 & 0 & 0 & 0 & N/A & N/A \\
\hline Caso & 0 & 0 & 1 & 1 & 0 & 12 & 3 & 0 & 0 & 0 & 0 & 0 & N/A & N/A \\
\hline
\end{tabular}


Bio - grafía. Escritos sobre la Biología y su Enseñanza. ISSN 2027-1034

Edición Extraordinaria. p.p. 1423 - 1432

Memorias del IX Encuentro Nacional de Experiencias en Enseñanza de la Biología y la Educación Ambiental. IV Congreso Nacional de Investigación en Enseñanza de la Biología.

\begin{tabular}{|c|c|c|c|c|c|c|c|c|c|c|c|c|c|c|}
\hline 10 & & & & & & & & & & & & & & \\
\hline $\begin{array}{c}\text { Caso } \\
11\end{array}$ & 0 & 1 & 1 & 1 & 0 & 11 & 2 & 0 & 1 & 0 & 0 & 0 & $\mathrm{~N} / \mathrm{A}$ & $\mathrm{N} / \mathrm{A}$ \\
\hline $\begin{array}{c}\text { Caso } \\
12\end{array}$ & 1 & 1 & 1 & 1 & 0 & 9 & 2 & 0 & 1 & 0 & 0 & 0 & N/A & $\mathrm{N} / \mathrm{A}$ \\
\hline $\begin{array}{c}\text { TOTA } \\
\text { L }\end{array}$ & 8 & $\begin{array}{l}1 \\
0\end{array}$ & 12 & 12 & 9 & $\mathrm{~N} / \mathrm{A}$ & $\mathrm{N} / \mathrm{A}$ & 1 & 2 & 0 & 1 & 0 & $\mathrm{~N} / \mathrm{A}$ & $\mathrm{N} / \mathrm{A}$ \\
\hline $\begin{array}{c}\text { TOTA } \\
\text { L } \\
\text { GLO } \\
\text { BAL }\end{array}$ & \multicolumn{7}{|c|}{51} & \multicolumn{7}{|c|}{4} \\
\hline
\end{tabular}

\section{ANALISIS DE RESULTADOS:}

En la tabla 1 se evidencia la comparación entre mapas conceptuales y cuestionaros como herramientas de evaluación, en donde, con la primera herramienta obtenemos un número elevado de obstáculos identificados, cincuenta y uno (51) en total; poniendo en evidencia a los obstáculos No se hace referencia a respiración celular y Desarticular la respiración de otros procesos celulares y del organismo como los que presentan la mayor frecuencia. además de poder evaluar tanto el número de conceptos usados para explicar el concepto de respiración como las relaciones inherentes entre ellos, poniendo en manifiesto la imagen de los conceptos y preposiciones que han apropiado en su estructura, (Novak y Gowin, 1999) características claves para evidenciar un aprendizaje significativo. Por su parte la herramienta de evaluación de cuestionario aporto un número mínimo de obstáculos identificados en el proceso de aprendizaje del concepto respiración, cuatro (4) en total; siendo Excesivo antropocentrismo el obstáculo que más frecuencia mostró, mientras que las columnas de "\#conceptos" y "conectores" se encuentran sin pertinencia para este tipo de herramienta. Lo cual deja sin evidencia si hay o no un aprendizaje significativo del concepto. Esta herramienta puede llegar a ser mal interpretada al mostrar pocos obstáculos presentes en el aprendizaje del concepto, esto se debe a la naturaleza de la herramienta la cual según Muñoz (2003), la de dar siempre una respuesta concreta sin tener en cuenta la estructura cognitiva previa del estudiante.

\section{CONCLUSIONES:}

En pro del aprendizaje del estudiante, se afirma que, para el proceso de enseñanza del concepto respiración es primordial, en primera medida identificar los posibles obstáculos para trabajar sobre ellos como eje central, lo que vuelve necesario revisar y replantear diferentes formas de evaluación en busca de pertinencia y precisión al identificar posibles obstáculos en la enseñanza de este concepto. Para este caso en particular resaltamos la necesidad de replantear el tipo de evaluación generada por el modelo de cuestionario, pues pasa por alto 
Bio - grafía. Escritos sobre la Biología y su Enseñanza. ISSN 2027-1034

Edición Extraordinaria. p.p. 1423 - 1432

Memorias del IX Encuentro Nacional de Experiencias en Enseñanza de la Biología y la Educación Ambiental. IV Congreso Nacional de Investigación en Enseñanza de la Biología.

gran cantidad de obstáculos los cuales repercuten en una baja calidad de la labor docente. El contraste entre las herramientas de evaluación, muestran claramente que los mapas conceptuales ofrecen mayor información del proceso de aprendizaje de los estudiantes detectando tanto obstáculos como fortalezas. El aprendizaje significativo aun cuando era el modelo pedagógico empleado por la institución, no era más que algo de carácter figurativo, pues se promueve en ella el uso de cuestionarios por sobre otras herramientas de evaluación.

\section{REFERENCIAS BIBLIOGRÁFICAS:}

Ahumada, P. 2005. La evaluación auténtica: un sistema para la obtención de evidencias y vivencias de los aprendizajes. Perspectiva Educacional, (45), 11-24. Disponible en: http://revele.com.veywww.redalyc.org/articulo.oa?id=333329100002 consultada el 01 de julio del 2016.

Ausubel, D. P. 2002. Adquisición y retención del conocimiento. Una perspectiva cognitiva. Barcelona: Paidós. 5, 6 y 7 de octubre de 2016. Ciudad Autónoma de Buenos Aires Argentina XII Jornadas Nacionales y VII Congreso Internacional de Enseñanza de la Biología Volver a las fuentes: La resignificación de la enseñanza de la Biología en aulas reales

Trillas.

Ausubel, D. P. 1976. Psicología educativa. Un punto de vista cognoscitivo. México:

Ausubel, D. P. 1973. La educación y la estructura del conocimiento. Buenos Aires: El Ateneo.

Curtis, H.; Barnes, S.; Schnek, A.; y Flores, G. 2006. Invitación a la Biología. 6ª ed. Buenos Aires: Médica Panamericana.

Orrego, M.; Dávila, A. y Tamayo, O. 2009. Obstáculos en el aprendizaje del concepto de respiración. Enseñanza de las Ciencias, Número Extra, VIII Congreso Internacional sobre Investigación en Didáctica de las Ciencias realizado en Barcelona: 1820-1822.

Mendonça, C. A. S., \& Silveira, F. P. R. D. A. 2012. Los Mapas Conceptuales Progresivos: un Estudio de los Estudiantes de la Escuela Primaria. Actas de la $5^{\mathbf{a}}$ Conferencia Internacional sobre Mapas realizada en La Valetta, Malta, septiembre de 2012

Monguí, C.C. 2014. Diseño de una herramienta para la enseñanza-aprendizaje de la respiración celular. Trabajo final, presentado como requisito para optar al título de Magister en la Enseñanza de las Ciencias Exactas y Naturales. Universidad Nacional de Colombia. 
Bio - grafía. Escritos sobre la Biología y su Enseñanza. ISSN 2027-1034

Edición Extraordinaria. p.p. $1423-1432$

Memorias del IX Encuentro Nacional de Experiencias en Enseñanza de la Biología y la Educación Ambiental. IV Congreso Nacional de Investigación en Enseñanza de la Biología.

Muñoz, T. G. 2003. El cuestionario como instrumento de investigación/evaluación. Disponible en: http://personal. telefonica. terra. es/web/medellinbadajoz/sociologia/ElCuestionario. Pdf consultado el 01 de julio del 2016.

Novak, J. D., Gowin, D. B., \& Otero, J. 1988. Aprendiendo a aprender. Barcelona: Martínez Roca.

Rodríguez Palmero, M. L. 2004. Teoría del aprendizaje significativo. Actas de la 1ra Conferencia Internacional sobre Mapas conceptuales realizada en Pamplona, España, septiembre de 2004. 